\title{
XIII.
}

\section{Zur Lehre von der multiplen selbständigen Gehirnnervenneuritis.}

(Fall von Diplegia facialis combinirt mit 0phthalmoplegia externa.)

Von

\section{Dr. med. Carl v. Rad, \\ Nervenarzt in Nürnberg.}

Fälle von neuritischer Gehirnnervenlähmung sind im Ganzen selten. Jeder einzelne neue Fall darf besonderes Interesse beanspruchen und der Veröffentlichung werth erscheinen. Am besten scheidet man die auf neuritischer Basis entstandenen Gehirnnervenlähmungen erstens in solche, die in Verbindung mit allgemeiner Polyneuritis vorkommen, und zweitens in die Formen, die ohne Betheiligung der spinalen Nerven als selbständige Affection der cerebralen Nerven auftreten.

Die Entstehungsursachen, welche zu einer Entzündung der Gehirnnerven führen, sind natürlich genau die gleichen, wie die, welche eine allgemeine multiple Neuritis der Extremitätennerven zur Folge haben. Traumatische, infectiöse, toxische und rheumatische Schädlichkeiten spielen hier wie dort die gleiche Rolle. Längst schon bekannt ist, dass die im Verlaufe der Diphtherie auftretende Polyneuritis mit Vorliebe neben den Extremitätennerven die Augen-, Rachen- und Kehlkopfmuskeln zu ergreifen pflegt. Von der der infectiösen Form der Polyneuritis nahestehenden Beri-Beri-Erkrankung-steht ebenfalls fest, dass sie Gehirnnerven sehr häufig befällt. Mit der erst in den letzten 2 Jahrzehnten verbesserten Kenntniss der neuritischen Erkrankungen wurde erst bekannt, dass die Gehirnnerren sich auch an dem entzündlichen Process betheiligen können.

Was zunächst die Fälle anbelangt, wo sich neben einer allgemeinen Neuritis eine solche eines oder mehrerer Gehirnnerven findet, so weist die Literatur, soweit sie mir zur Verfügung stand, zahlreiche Beispiele dafür auf. Da in der Arbeit von Minkowski ${ }^{1}{ }^{*}$ ) die vor 1888 veröffentlichten Fälle aufgeführt sind, so berücksichtigte ich hauptsächlich nur die im Laufe der letzten 10 Jahre erschienenen Arbeiten. Wenn ich von der im Verlaufe einer Polyneuritis auftretenden Neuritis optica

*) Literatur s. am Schluss d. Art.

Deutsche Zeitschr. f. Nervenheilkande. XVII. Bd. 
oder retrobulbaris absehe, so fällt bei dex Durchsicht der Literatur auf, dass am allerhäufigsten der Nervus facialis ein- oder besonders doppelseitig erkrankt ist. Derartige Fälle wurden beschrieben von Pierson ${ }^{2}$ ), Eisenlobr ${ }^{3}$ ), Oppenheim ${ }^{4}$ ), Strube ${ }^{5}$ ), Buzzard ${ }^{6}$, Savage ${ }^{7}$, Targow ${ }^{8}{ }^{8}$, , Westphalen ${ }^{9}$ ), Crocq ${ }^{10}$ ), Stieglitz ${ }^{11}$ ) und 2 mal von v. Strümpell12) u. 13). Betheiligung des Opticus ein- oder beiderseitig konnten unter Anderen nachweisen Eichhorst ${ }^{14}$ ), Löwenfeld ${ }^{15}$ ), Remak ${ }^{16}$ ), Lilienfeld ${ }^{17}$ ), Thomsen ${ }^{18}$ ), Kender ${ }^{19}$ ), Miura ${ }^{20}$ ), Goldscheider ${ }^{21}$ ), Fuchs ${ }^{22}$ ), Jolly ${ }^{23}$ ) und Schanz ${ }^{24}$ ).

Ueber eine Betheiligung von motorischen Augennerven in Fällen von Polyneuritis berichteten Hiller ${ }^{25}$ ), Boeck ${ }^{26}$ ), Pal 27 ), Schulz ${ }^{28}$ ), Lilienfeld ${ }^{29}$ ) und Jolly ${ }^{30}$ ). In dem Falle des letzterwähnten Forschers waren neben beiden Abducentes noch beide Optici und Faciales ergriffen. Mehrere Autoren, wie Leyden ${ }^{31}$, Sorgo ${ }^{32}$ ), Oppenheim ${ }^{33}$ ), Achard und Soupault ${ }^{34}$ ) beschrieben ein Mitergriffensein der Nn. vagi; es ist das eine äusserst ernste und lebensgefährliche Complication der Neuritis. Achard und So npault konnten in ihrem Falle die neuritische Degeneration des Nerven anatomisch nachweisen. Ueber eine Betheiligung beider Nn. acustici berichtete Strümpell ${ }^{12}$ ). Es kam in seinem Falle von alkoholischer Polyneuritis neben einer Diplegia facialis zum Auftreten von beiderseitiger, rein nervöser Taubheit, die nach 3 Wochen vorüberging, um einem lange andauernden Ohrensausen Platz zu machen (Neuritis acustica). Während die neuritische Gehirnnervenlähmung in der Regel sich auf einen oder wenige Nerven zu beschränken pflegt, konnten Roth ${ }^{25}$ ) und Schlier ${ }^{36}$ ) eine Affection mebrerer Gehirnnerven nachweisen. In dem Roth'schen Falle waren 6 verschiedene Nerven neuritisch afficirt, in dem durch seinen Verlauf und seine häufigen Recidive interessanten Fall von Schlier waren neben den Nerven der Arme und Beine der Opticus, Abducens und Facialis der linken Seite, sowie der N. vagus ergriffen.

Ebenfalls um eine Lähmung mehrerer Gehirnnerven handelt es sich in dem von Rossolimo ${ }^{37}$ ) beschriebenen Falle. Bei seinem Patienten hatte sich auf luetischem Boden und nach einer Erkältung eine multiple Neuritis entwickelt, welche ausser einer Reihe von peripherischen Spinalnerven die ersten Aeste der beiden Trigemini und die beiden $\mathrm{Nn}$. oculomotorii und trochleares betraf. Die Function der inneren Aeste beider Oculomotorii blieb völlig erhalten. Während die Lähmungen der genannten Nerven völlig verschwanden, entwickelte sich später bei dem Patienten eine Tabes mit Myosis und Lichtstarre. Dammron-Meyer ${ }^{38}$ ) beobachteten im Verlaufe einer Polyneuritis das Auftreten mehrerer Augenmuskellähmungen und waren im Stande, ihre klinische Diagnose durch die anatomische Untersuchung zu bestätigen. 
Auf Bleivergiftung konnte Mannaberg ${ }^{39}$ ) seine 2 Fälle von Polyneuritis zurückführen. Neben einer Affection an den Armen und Beinen fand sich im ersten eine beiderseitige Neuritis optica, totale rechtsseitige Oculomotorius- und Facialislähmung. In seinem 2. Falle waren ausser beiden Optici der rechte Abducens und der linke Facialis ergriffen. Ich selbst hatte Anfang dieses Jahres Gelegenheit, 2 in diese Gruppe gehörige Fälle zu untersuchen. Bei einem 47jährigen Manne kam es zu einer Polyneuritis beider Arme und Beine, die sich mit einer completen Ophthalmoplegia externa und Reizerscheinungen im Bereiche des linken ersten Trigeminusastes verband. In Laufe der Behandlung erfuhren sowohl die Lähmung der Augenmuskeln, als auch die schmerzhafte Schwäche der Beine eine wesentliche Besserung. Einen 2. Fall hatte ich Gelegenheit im hiesigen städtischen Krankenhause zu untersuchen. Der Fall ist kurz folgender: Bei einem jungen Mann entwickelte sich neben einer multiplen Neuritis der Arme und Beine eine nur kurz bestehende Diplegia facialis, zu der sich im weiteren Verlauf eine Neuritis des linken Opticus und eine Schwäche der Bulbärnerven gesellte, die eine Erschwerung der Sprache und des Schluckactes zur Folge hatten. Der Fall verlief sehr günstig.

Während die in Verbindung mit Entzündung der Extremitätennerven auftretende Gehirnnervenneuritis schon früher zur Beobachtung kam, wurde das selbständige Vorkommen einer cerebralen entzündlichen Erkrankung der Gehirnnerven ohne Betheiligung der spinalen mit wenigen Ausnahmen meist erst in den letzten Jahren beschrieben.

Fälle von uncomplicirter ein- oder doppelseitiger Facialislähmung auf neuritischer Basis beobachteten Althaus ${ }^{40}$ ), Bregmann ${ }^{41}$ ) und Déjèrin $\mathrm{e}^{42}$ ). In dem Hoffmann'schen ${ }^{43}$ ) Falle kam es neben einer Diplegia facialis zu einer Neuritis beider Optici und Acustici. Kauf$\left.\operatorname{mann}{ }^{44}\right)$ beschrieb eine neuritische Lähmung des linken Facialis und linken Abducens, sowie des zweiten Astes des linken Trigeminus. Im Verlaufe einer Leukämie konnte Eisenlohr ${ }^{45}$ ) eine acute multiple Neuritis der Bulbärnerven beobachten. Ein weiteres interessantes Beispiel für das Auftreten der Gehirnnerrenneuritis ist die Beobachtung von Moebius s6). Bei einem 20jährigen Manne hatte sich in Folge einer unzweifelhaften Errkältung im Eisenbahncoupé gleichzeitig mit starken reissenden Schmerzen in der rechten Seite des Gesichts, welche nachher in eine Hautanästhesie des genannten Theiles übergegangen sind, eine vollständige Lähmung aller äusseren Muskeln des rechten Auges entwickelt, während die Accomodation und Pupillenreaction intact geblieben sind. 
Möbius führt die Lähmung des rechten Trigeminus und die Ophthalmoplegia externa auf eine rheumatische Neuritis zurück.

Ueber das Befallensein zahlreicher Gehirnnerven berichtet uns die Publication von v. Hösslin ${ }^{47}$ ).

Es waren betroffen rechts der Nerrus trigeminus und abducens, die Chorda tympani und das Gaumensegel; auf der linken Seite der Opticus, Trigeminus, Abducens, Facialis und Hypoglossus. Gleichzeitig bestanden Reizerscheinungen von Seiten des Nervus vagus. Der Verlauf der Erkrankung war ein äusserst günstiger, indem nur die Erblindung des linken Auges bestehen blieb und alle anderen Lähmungen sich völlig zurückbildeten.

Besondere Beachtung verdient ferner die Abhandlung von Fla$\left.\tan ^{48}\right)$, der eine Neuritis des Facialis und Acusticus beobachten und durch die anatomische Untersuchung bestätigen konnte. Ebenso konnten Gibson und Turner ${ }^{49}$ ) in ihrem Falle von linksseitiger Oculomotoriuslähmung post mortem eine hämorrhagische Neuritis nachweisen.

Einige Fälle fand ich in der Literatur beschrieben, wo die Möglichkeit einer neuritischen Genese der Lähmungen zwar nicht ausdrücklich erwähnt, aber doch nicht ganz von der Hand zu weisen war. So beschreibt Mendel ${ }^{50}$ ) ein en Fall von multipler Hirnnervenlähmung; die er beim vollständigen Fehlen aller sonst dafür verantwortlich zu machenden Ursachen als eine rheumatische bezeichnet. Betroffen waren der linke Oculomotorius und Facialis. Der Verlauf war ein sehr günstiger. Beachtenswerth ist, dass der Lähmung Schmerzen und allgemeines Uebelsein vorausgingen.

Aus dem gleichen Jahre stammt eine Veröffentlichung von Uhthoff ${ }^{51}$ ), der drei Fälle von doppelseitiger Accomodationslähmurıg in Folge ron Influenza beschreibt.

Der eine Fall, auf den ich besonders hinweisen möchte, war complicirt durch eine Ophthalmoplegia externa und Parese der Schlundmuskeln und des Gaumensegels. Heilung trat nach wenigen Wochen ein. Uhth off weist auf die Aehnlichkeit der beschriebenen Erscheinungen mit denjenigen bei postdiphtheritischer Lähmung hin.

Bunzel ${ }^{2}$ ) beschreibt endlich noch eine Lähmung der Augenmuskeln bei Polyarthritis. Da mir die Arbeit nur im Referat zugänglich war, vermag ich nichts Näheres darüber anzugeben. Jedoch liegt bei den innigen Beziehungen, die zwischen der arthritischen Diathese und der Neuritis bestehen, die Möglichkeit einer neuritischen Entstehung der Lähmungen nahe.

Nach dieser Literaturübersicht, die auf Vollständigkeit keinen Anspruch machen kann, da mir die einzelnen Zeitschriften und Ab- 
handlungen nur in beschränktem Maasse zugänglich waren, möchte ich nun über einen selbst beobachteten Fall berichten, der auf gewisses Interesse Anspruch machen darf. Er ist ausgezeichnet durch eine auf neuritischer Basis entstandene Diplegia facialis, zu der sich eine beiderseitige Ophthalmoplegia externa mit Freibleiben des Levator palpebrae gesellt.

Der Fall ist kurz folgender:

B. E., 14jähriger Kaufmannslehrling, stammt aus einer durchans gesunden, mit Nervenkrankheiten nicht belasteten Familie. Mit 8 Jahren litt er, wie aus einem Bericht des damals behandelnden Arztes zu ersehen ist; an einer sehr schweren tuberculösen Cerebrospinalmeningitis, die wider Erwarten in Genesung ausging. Seit jener Erkrankung will Pat. das linke Auge nicht mehr völlig schliessen können und bleibe beim Schlafen eine kleine Spalte offen. Sonst sei nicht die geringste Störung in der Beweglichkeit der linken Gesichtshälfte zurückgeblieben. (Die Angaben des Pat. und die seiner Eltern gehen über diesen Punkt recht anseinander.) Ende Mai 1899 traten ohne nachweisbare Ursache plötzlich sehr heftige Schmerzen hinter beiden Ohren auf, die in beide Gesichtshälften ausstrahlten. Etwa 14 Tage darauf bemerkte Pat. eine sehr stark behinderte Beweglichkeit beider Gesichtshälften. Augenschiuss, Stirnrunzeln, Lachen, Pfeifen, Verziehen des Mundes wurden unmöglich. Auch stellte sich Doppeltsehen beim Blick nach rechts aussen ein. Pat. begab sich deshalb in die Behandlung des Augenarztes Herrn Dr. v. Forster, welcher die grosse Freundlichkeit hatte, mir den Fall zur weiteren Behandlung zu überweisen. Es ist mir eine angenehme Pflicht, Herrn Dr. v. Forster bestens dafür zu danken.

Bei der am 20. Juni 1899 vorgenommenen Untersuchung ergab sich folgender Befund:

Es besteht beiderseitige Facialislähmung mit Betheiligung sämmtlicher Aeste. Am stärksten sind die Stirnangenäste, am schwächsten die Unterkieferäste befallen.

Das Gesicht ist ausgesprochen maskenartig, völlig starr, jede minische Bewegung in demselben ist erloschen. Die reflectorische Erregbarkeit ist völlig aufgehoben. Druck auf die Austrittsstellen beider Nn. faciales hinter den Ohren am Foramen stylomastoideum sowie an den einzelnen elektrischen Reizpunkten der Nerven wird als sehr schmerzhaft empfunden.

Die schon normaler Weise bestehende Empfindlichkeit bei elektrischer Reizung des Facialis ist so sehr gesteigert, dass die elektrische Untersuchnng äusserst schmerzhaft und sehr erschwert ist. (Eine Anschwellung der Nerven ist nirgends fühlbar.)

Die mechanische Erregbarkeit der Gesichtsmuskeln ist gesteigert. Das Beklopfen derselben wird als schmerzhaft angegeben.

Die elektrische Untersuchung ergiebt deutliche Entartungsreaction, die Erregbarkeit vom Nerven aus ist fast erloschen, bei directer galvanischer Reizung erfolgen träge Muskelznckungen und überwiegt die ASZ über die KSZ. Mit dem faradischen Strom sind die Muskeln nicht erregbar.

Gaumensegel und Geschmack sind völlig intact.

Es besteht keine Hyperacusis. 
Im Bereiche des Trigeminus keine Störung.

Angenbefund. Es besteht rechtsseitige Abducenslähmung mit den dafür charakteristischen Doppelbildern.

Die übrigen Augenbewegungen sind frei.

Die Pupillen sind gleich und von mittlerer Weite, reagiren gut anf Licht und Convergenz.

Augenhintergrund normal bis auf etwas stärker gefiillte Venen.

Die Untersuchung des übrigen Nervensystems ergiebt normale Verhältnisse. Von Seiten der Extremitätennerven bestehen keinerlei krankhafte Erscheintungen.

Die Untersuchung der Brnst- und Bauchorgane ergiebt ausser einer leichten Abschwächung des Schalles auf der linken Lungenspitze beim Fehlen anscultatorischer Erscheinungen durchaus normale Verhältnisse.

Der Urin ist frei von Eiweiss and Zucker.

Der weitere Verlanf gestaltete sich folgendermassen: Die Diplegia facialis blieb vorerst unverändert bestehen, dagegen trat am 25. Juni eine Lähmung des linken Abducens auf; zu der sich am 27. Juni eine solche beider Mm. interni, sowie beider Nn. trochleares gesellte. Es kam also zu einer Ophthalmoplegia externa. Die Beweglichkeit beider Bulbi war nach allen Richtungen hin sehr beträchtlich eingeschränkt. Der Levator palpebrae blieb beiderseits frei, die Reaction auf Licht, sowie die Möglichkeit, zu accomodiren, blieben erhalten. Von den äusseren Augenmuskeln waren am stärksten diojenigen betroffen, die den Bulbus um die verticale Axe drehen. Von den um die horizontale Axe drehenden Muskeln waren die Senker stärker paretisch als die Heber.

Mitte Jnni besserte sich die Excursionsfähigkeit der Bulbi etwas, namentlich functionirten die Mm. interni besser. Die sehr lästigen Doppelbilder bestanden noch fort.

In der beiderseitigen Mundmusculatur wurde die Beweglichkeit etwas besser, die übrigen vom Facialis versorgten Muskeln blieben noch völlig starr. Es bestand ausgesprochene Entartungsreaction. Die Schmerzhaftigkeit der Nerven und Muskeln wurde geringer.

Ende Juni war die Beweglichkeit der Bulbi eine weit bessere geworden, die Heber und Senker, sowie die Einwärtswender waren fast normal, eine nennenswerthe Behinderung der Beweglichkeit bestand nur mehr in den Mm. externi.

Die Beweglichkeit des Hundes war ebenfalls eine weit bessere geworden, namentlich auf der rechten Seite, sonst blieb der Befund unverändert. Es bestand noch völlige Entartungsreaction, nur im Bereiche des Mundfacialis wurden die Zuckungen etwas besser, ohne jedoch ihren trägen Charakter ganz zn verlieren. Die Schmerzhaftigkeit der Nerven auf Druck war nun fast völlig verschwunden.

Mitte August waren die Augenbewegungen ganz frei, die Doppelbilder bestanden noch fort. Die Beweglichkeit des Gesichtes hat sich auch gebessert. Völlig unbeweglich war nur mehr die Stirn.

Pat. konnte rechts lachen und gut den Mund verziehen, links nur andeutungsweise. Bei galvanischer Reizung des Nerven liess sich beiderseits eine schwache, aber doch deutliche Zuckung auslösen, bei directer Reizang zeigten die Muskeln nicht mehr so trägen Charakter und war die $\mathrm{KSZ}=$ ASZ. Faradisch waren die Muskeln weder direct noch indirect erregbar. 
Erst Anfang September trat nun eine wesentliche Besserung der Beweglichkeit der gesammten Gesichtsmusculatur ein, die mit einer Besserung der elektrischen Erregbarkeit einherging.

Bei Zunahme der direeten and indirecten galvanischen Erregbarkeit waren auch mit dem faradischen Strom schwache Muskelzuckungen auszulösen.

Im weiteren Verlauf trat eine stetig zunehmende Besserung ein, so dass Anfang October Pat. als völlig geheilt betrachtet werden konnte.

Am 10. October konnte ich folgenden Befund aufnehmen:

Die Augenbewegungen sind völlig frei. Es bestehen keine Doppelbilder mehr. Die Gesichtsmuskeln sind beiderseits recht gut beweglich. Pat. vermag alle ihm aufgegebenen Bewegungen, wie Augenschliessen, Naserümpfen, Zähnezeigen, Lachen, den Mund nach der Seite verziehen, prompt und gut auszuführen. Seine Gesichtsbewegungen unterscheiden sich in nichts mehr von denen eines Gesunden; nur klagt er über ein bei denselben auftretendes leichtes Gefühl von Spannung im Gesicht.

Bei der elektrischen Untersuchung lassen sich vom Nerven aus mit beiden Stromesarten schwache, aber doch keineswegs träge Muskelzuckungen auslösen. Bei Anwendung des galvanischen Stromes fallen die Zuckungen besser aus als bei Reizung mit dem faradischen. Die directe Muskelerregbarkeit ist für beide Stromesarten noch etwas herabgesetzt, doch erfolgen überall kurze, kräftige Zuckungen, die nicht mehr wie früher einen trägen, wurstförmigen Verlauf zeigen, auch überwiegt die KSZ über die ASZ.

Die Behandlung bestand in Anfangs täglich, später jeden zweiten Tag. vorgenommener Galvanisation der Nn. faciales und denen ihnen versorgten Muskeln.

Resumiren wir kurz die wichtigsten Punkte des Krankheitsbildes, so sehen wir bei einem 14 jährigen jungen Mann, der aus nicht belasteter Familie stammt und ausser einer tuberculösen Cerebrospinalmeningitis stets gesund war, ohne nachweisbare Ursachen plötzlich sehr heftige, von beiden Ohren in die Gesichtshälften ausstrahlende Schmerzen auftreten, denen nach 14 Tagen eine complete Lähmung beider Facialisnerven sich anschloss. Fast gleichzeitig mit der Diplegia facialis entwickelte sich eine rechtsseitige Abducenslähmung, zu der sich nach wenigen Tagen in rascher Aufeinanderfolge der einzelnen Muskeln eine Lähmung sämmtlicher äusseren Augenmuskeln, die nur den Levator palpebrae verschont liess, gesellte. Es kam also zu einer completen Ophthalmoplegia externa bei gut erhaltener Lichtreaction und Accomodationsfähigkeit. In hohem Grade beachtenswerth war die ausgesprochene Schmerzhaftigkeit beider $\mathrm{Nn}$. faciales sowohl an ihren Austrittsstellen hinter dem Ohr, als auch in ihrer ganzen Ausbreitung im Gesicht. Die spontan durch Druck noch zu steigernden Schmerzen waren sehr heftige.

Die Lähmung trug einen ausgesprochen peripheren Charakter, umfasste sämmtliche Aeste der Nerven und ging mit Entartungsreaction einher. Der weitere Verlauf der Erkrankung war ein ausserordentlich 
günstiger. Zuerst besserte sich die Lühmung der Augenmuskeln und erst nach 3 Monaten die der beiden Gesichtsnerven. Nach $4 y_{2}$ Monaten war der ganze Process abgelaufen und völlige Heilung eingetreten.

Die Diagnose bot im vorliegenden Falle keine besonderen Schwierigkeiten. Das acute, fast apoplectische Auftreten der bilateral einsetzenden Lähmungen, wie ganz besonders die initialen Schmerzen und die sehr starke Druckschmerzhaftigkeit der Nerven und der von ihnen versorgten Muskeln, sowie der ausgesprochen periphere Charakter der Lähmung sprachen mit grosser Sicherheit für eine neuritische Affection.

Die weitere klinische Beobachtung, in deren Verlauf völlige Heilung eintrat, konnte die gestellte Diagnose vollauf bestätigen.

Die einzelnen krankhaften Erscheinungen lassen sich ungezwungen durch die Annahme einer entzündlichen Nervenaffection erklären. Die neuritischen Cardinalsymptome waren namentlich während der Entwicklung des Leidens voll ausgeprägt vorhanden. Erhebliche spontane Schmerzen im Gesicht leiteten den Process ein, zu denen sich bald eine beträchtliche Druckschmerzhaftigkeit im Bereiche der Gesichtsäste der Nn. faciales gesellte. Das Auftreten von Schmerzen in einem so rein der motorischen Leitung dienenden Nerven, wie dem Facialis, ist wiederholt schon beobachtet worden. Es handelt sich eben um eine Reizung der Nervi nervorum. Die centripetale Leitung des Schmerzes erfolgt nach Remak wohl durch Anastomosen mit dem N. trigeminus.

Dass die elektrische Reizung des entzündeten Facialis äusserst schmerzhaft ist, liegt auf der Hand. In manchen in ihrer Genese zweifelhaften Fällen von Facialislähmung mag vielleicht eine sehr beträchtlich gesteigerte Schmerzhaftigkeit der elektrischen Untersuchung einen Fingerzeig für die Natur des Processes abgeben, zumal wenn es sich um Formen handelt, in denen die übrigen neuritischen Symptome nur schwach oder gar nicht ausgeprägt sind.

Die Lähmung selbst war ausgezeichnet durch das rasche, acute Einsetzen, das ziemlich gleichmässige Befallensein aller Aeste, das Erloschensein aller reflectorischen Bewegungen im Bereiche der gelähmten Muskeln und durch die degenerativen Veränderungen der Muskeln selbst.

Die Veränderungen der elektrischen Erregbarkeit, welche der Lähmung auf dem Fusse folgten, bestanden in einer deutlichen Entartungsreaction mit zeitweiligem Verlust jeder indirecten Erregbarkeit für beide Stromesarten. Ein Stadium einer initialen gesteigerten Nervenerregbarkeit kam nicht zur Beobachtung. Nach dem elektrodiagno- 
stischen Befund musste die Lähmung der Gesichtsnerven als schwere bezeichnet werden. Mit Zunahme der regenerativen Vorgänge besserte sich auch allmählich die elektrische Erregbarkeit. Nachdem ganz langsam die Nervenerregbarkeit (und zwar zuerst die für den galvanischen Strom) zurückgekehrt war, verschwand auch nach und nach die pathologische Reaction des Muskels. Doch muss im Auge behalten werden, dass die Restitution der elektrischen Erregbarkeit wie fast stets eine viel langsamere war, als diejenige der motorischen Function.

Das Symptom der Anschwellung des entzündlich erkrankten Nerven wurde im vorliegenden Falle völlig vermisst. Bei der recht geschïtzten Lage der Gesichtsnerven war es auch nicht zu erwarten.

Beachtenswerth ist ferner noch der Umstand, dass bei dem $\mathrm{Pa}$ tienten im Anschluss an die tuberculöse Meningitis, die er vor sechs Jahren durchmachte, eine leichte Parese des 1. Orbicularis zurückgeblieben war. Es ist also der linke Facialis zweimal von einer Lähmung befallen worden. Interessant ist nun, dass die im Anschluss an die zweite Lähmung eingetretenen regenerativen Veränderungen auch die Fasern des Augenfacialis wieder functionstüchtig machten, die bei der ersten Erkrankung lange Jahre geschädigt blieben.

Was wun den Augenbefund anbelangt, so baben wir hier das Bild einer Ophthalmoplegia completa mit Verschontbleiben des Levator palpebrae vor uns. Accornodation und Lichtreaction blieben völlig intact. Die Lähmung aller äusseren Augenmuskeln, die eine völlige Unbeweglichkeit der Bulbi zur Folge hatte, ist zuräckzuführen auf eine Affection einzelner Fasern der beiden Oculomotorii, sowie beider Nn. abducentes und trochleares. Ueber die Möglichkeit des Auftretens einer partiellen Lähmung des Oculomotorius, welche bei Erhaltang der Function der inneren Angenmuskeln nur die die äusseren innervirenden Faserbündel des Nerven betrifft, ist von ophthalmologischer und neurologischer Seite schon viel discutirt worden. Während Mauthner hauptsächlich die Meinung vertritt, dass eine derartige Differenzirung der Symptome bei Weitem leichter ihre Erklärung in der Annahme einer nuclearen Affection finde, sprechen andere Forscher dieses Vorkommen auch peripheren Affectionen des Nerven zu. Ich glaube, dass mein Fall zur Stütze der letzteren Anschaung verwerthet werden darf.

Ganz abgesehen von der gleichzeitig in Erscheinung getretenen zweifellos neuritischen Diplegia facialis spricht schon das so acute bilaterale symmetrische Einsetzen der Lähmungen und das völlige Verschwinden derselben innerhalb kurzer Zeit gegen das Bestehen einer nuclearen Affection. 
Irgend welche cerebralen Allgemeinerscheinungen, die bei Herden in der Kernregion der Gehirnnerven ja doch auf die Dauer nie fehlen, kamen auch nie zur Beobachtung. Es lässt also der ganze Verlauf und insbesondere die Rücksicht auf die gleichzeitige neuritische Gesichtslähmung keine andere Deutung zu, als dass die Ophthalmoplegia externa auf eine neuritische Affection der motorischen Augennerven zurückzuführen ist. Dieses elective Verhalten der Lähmung des Oculomotorius ist durch die Annahme einer neuritischen Affection gut zu erklären. Denn gerade bei der Neuritis von Spinalnerven sehen wir oft diese Differenzirung der Lähmungssymptome auftreten, so dass oft bald mehr oder weniger, ja oft ausschliesslich die motorischen oder sensiblen Fasern eines Nerven erkrankt sind.

Von den eventuell differentialdiagnostisch in Betracht kommenden Erkrankungen wäre in erster Linie zu erwähnen eine luetische Basalmeningitis oder specifische Neuritis der Gehirnnerven. Ganz abgesehen davon, dass die Anamnese keinerlei Anhaltspunkte für eine hereditäre Lues und nur um eine solche könnte es sich handeln, ergiebt, spricht schon das völlige Fehlen aller cerebralen Erscheinungen, die wir bei diesen luetischen Erkrankungen nie vermissen, dagegen. Wenn anch im Verlaufe der Hirnlues Schwankungen häufig vorkommen, so ist doch eine völlige Heilung ohne jede specifische Therapie ein äusserst seltenes Vorkommniss.

Gegen basale Tumoren, die ja ebenfalls multiple Hirnnervenlähmungen zur Folge haben können, spricht in erster Linie der günstige Verlauf, auch müsste in einem solchen Falle der hart neben dem Facialis liegende $N$. acusticus mitbetroffen sein; auch wäre es dann schwer zu verstehen, wie der zwischen den befallenen Nerven liegende $\mathrm{N}$. trigeminus hätte frei bleiben können.

Die Aetiologie ist mir im vorliegenden Falle unklar. $\mathrm{Ob}$ der tuberculösen Diathese irgend welche ursächliche Bedeutung zugemessen werden darf, möchte ich unentschieden lassen. Im Allgemeinen sehen wir die tubereulöse Neuritis meist nur bei sehr heruntergekommenen, kachektischen Individuen auftreten. Sie ist dann mehr eine Folge des Marasmus, als der Infectionskrankheit selbst. Diphtherie muss ebenfalls ausgeschlossen werden. Da auch keine nachweisbare Erkältung vorausgegangen ist, muss die Frage nach der Entstehungsursache offen gelassen werden.

Die Prognose der Gehirnnervenneuritis entspricht im Allgemeinen natürlich derjenigen der allgemeinen Polyneuritis. Sie ist abhängig von der besonders nach dem elektrodiagnostischen Befund zu beurtheilenden Schwere der degenerativen Veränderungen.

Im Grossen und Ganzen glaube ich, darf man die Prognose in 
solchen Fällen günstig stellen. Die Mehrzahl der in der Literatur beschriebenen Fälle nahm einen günstigen Verlauf. Eine Ausnahme machen natürlich die Fälle, die mit einer Erkrankung des N. vagus einhergehen. Jedenfalls bietet die multiple Gehirnnervenlähmung auf neuritischer Basis gegenüber allen anderen Erkrankungen, welche zu einer Lähmung mehrerer Gehirnnerven führen, die weitaus beste Prognose. Die Möglichkeit eines Recidivs muss natürlich im Auge behalten werden.

Die Therapie wird meist eine rein symptomatische sein müssen. Gegen die initialen Schmerzen werden feuchtkalte oder feuchtwarme Umschläge empfohlen. Mir hat sich als schmerzlindernd sehr die Anodengalvanisation bewährt.

Die Hauptsache bleibt natürlich eine regelmässig und längere Zeit fortgesetzte elektrische Behandlung der gelähmten Muskeln und Nerven. Am meisten möchte ich den galvanischen Strom empfehlen, der faradische ist namentlich im Anfangsstadium zu schmerzhaft und, so lange bei faradischer Reizung keine Zuckung ausgelöst werden kann, ist auch eine faradische Behandlung zwecklos. Sobald eine Spur von Beweglichkeit vorhanden ist, veranlasse ich meine Kranken mit Gesichtslähmung selbständig vor dem Spiegel Bewegungsversuche und Uebungen anzustellen.

\section{Literatur:}

1) Minkowski, Beiträge zur Pathologie d. multiplen Neuritis. Mittheilungen aus der Königsberger Klinik.

2) Pierson, Volkmann'sche Hefte. 1882. Nr. 229.

3) Eisenlohr, Berl. klin. Wochenschr. 1887. Nr. 42.

4) Oppenheim, Berl. klin. Wochenschr. 1887. Nr. 17.

5) Strube, I.D. Berlin 1881.

6) Buzzard, Lancet 1885.

7) Savage, Revue de médecin. 1891, Februar.

8) Targowla, Revue neurol. 1894. Nr. 16.

9) Westphale n, Petersburger Wochenschr. 1890. Nr. 21.

10) Crocq, Journ. de neur. et d'hypnot. 1898. Nr. 10.

11) Stieglitz, Boston med. and surg. journ. 1898. Nr. 3.

12) v. Strümpell, Neurol. Centralblatt 1889.

13) Derselbe, Deutsch. Archiv f. klin. Medicin 1899.

14) Eichhorst, Virchow's Archiv. 1877.

15) Löwenfeld, Bayr. ärztl. Intelligenzblatt 1885.

16) Remak, Neurol. Centralblatt. 1885. Nr. 14.

17) Lilienfeld, Berl. klin. Wochenschr. 1885. S. 757.

18) Thomsen, Archiv f. Psych. 1890.

19) Kender, The American Lancet. 1890, Dec. 
220 XIII. v. RAD, Zur Lehre von der multiplen selbständigen Gehirnnervenneuritis.

20) Miura, Berl. klin. Wochenschr. 1891. Nr. $3 \overline{\text { T }}$

21) Goldscheider, Charité-Annalen. 1892.

22) Fuchs, Deutsche Zeitschrift f. Nervenheilkunde. 1893. Heft 4.

23) Jolly, Berl. klin. Wochenschr. 1894. S. 283.

24) Schanz, Deutsche medic. Wochenschr. 1896. Nr. 28.

25) Hiller, Berl. klin. Wochenschr. 1881. Nr. 41.

26) Boeck, Tidskrift für prakt. Mediz. 1885. Referat: Neurol. Centralblatt. 1885.

27) Pol, Ueber multiple Neuritis. Wien 1891.

28) Schulz, Neurol. Centralblatt 1885.

29) Lilien feld, Berl. klin. Wochenschr. 1885. S. 727.

30) Jolly, Berl. klin. Wochenschr. 1884. S. 283.

31) Leyden, Zeitschrift f. klin. Med. 1. Band.

32) Sorgo, Ztschr. f. klin. Medic. 32. Band.

33) Oppenheim, Berl. klin. Wochenschr. 1890. Nr. 24.

34) Achard u. Soupault, Archiv de méd. exper. 1893. Nr. 13.

35) Roth, cit. bei v. Hösslin, Münch. med. Wochenschr. 1896.

36) Schlier, Ztschr. f. klin. Medic. 37. Band.

37) Rossolimo, Neurol. Centralblatt. 1890.

38) Dammron-Meyer, Bull. méd. 1888.

39) Manaberg, Neurol. Centralblatt. 1897.

40) Althaus, The Brit. med. journ. 1890.

41) Bregmann, Refer. Virchow-Hirsch 1895.

42) Déjerine, Revue de médic, 1898. Nr. 1.

43) Hoffman n, Arehiv. f. klin. Medic. 1894.

44) Kaufmaun, Zeitschr. f. Ohrenheilkunde. 1898. XXX.

45) Eisenlohr, Virchow's Archiv. 1878.

46) Moebius, Centralblatt f. Nervenheilkunde. 1886.

47) v. Hösslin, Münch. med. Wochenschr. 1896.

48) Flatau, Zeitschr. f. klin. Medic. Bd. 32.

49) Gibs on u. Turner, Edinb. méd. journ. 1898, Mai.

$50)$ Mendel, Neurol. Centralblatt 1890.

51) Uhth off, Deutsche medic. Wochenschr. 1890.

52) Bunzel, Prag. med. Wochenschr. 1896, April. 